\title{
Provisioning of Street Lighting based on Ambience Intensity for Smart City
}

\author{
Rajina R Mohamed ${ }^{1}$, Mohamad A Mohamed ${ }^{2}$, Arniyati Ahmad ${ }^{3}$, Muhamad A H Abd Halim ${ }^{4}$ \\ ${ }^{1}$ College of Computer Science and Information Technology, Universiti Tenaga Nasional, Malaysia \\ ${ }^{2}$ Faculty of Informatics and Computing, Universiti Sultan Zainal Abidin, Besut Campus, Malaysia \\ ${ }^{3,4}$ Faculty Science and Technology Defence, Universiti Pertahanan Malaysia, Sg. Besi Camp, Malaysia
}

\begin{tabular}{l}
\hline Article Info \\
\hline Article history: \\
Received Sep 30, 2018 \\
Revised Oct 24, 2018 \\
Accepted Nov 3, 2018 \\
\hline
\end{tabular}

\section{Keywords:}

Ambient Intensity

Energy Efficient

Energy Savings

Smart City

Street Lighting

\begin{abstract}
Street lighting is a component that require an efficient energy management system for comprehensive energy savings. It should provide ultimate comfort and safety to road users that contribute to the stability of economy and social of the country as well to the smart city development. Currently many street lighting especially in Malaysia, which is ON in the evening and OFF before the sun sets without taking into consideration the ambient brightness and also the number of vehicles. This condition may increase the use of energy as well as dangerous to motorist. Thus this Ambient Street Lighting project was developed using embedded-based Arduino microcontroller for lighting management based on the circumstances surrounding of the road. A threshold value is set based on a reading of the actual situation in order to test the functionality of the prototype. The Ambient Street Lighting prototype have reacted with success towards surrounding brightness/darkness and number of cars passing by on the street.
\end{abstract}

Copyright $(0) 2018$ Institute of Advanced Engineering and Science. All rights reserved.

\section{Corresponding Author:}

Rajina R Mohamed,

College of Computer Science and Information Technology,

Universiti Tenaga Nasional, Malaysia.

Email: rajina@uniten.edu.my

\section{INTRODUCTION}

Internet of Things (IoT) is an evolution to a situation where many devices can be controlled remotely and automatically. IoT has a dominant impact for energy efficiency and organized energy management. Organized energy management can be achieved through smart management system. Street lighting requires an efficient energy management system for comprehensive energy savings but providing ultimate comfort and safety to road users that contribute to the stability of economy and social of the country towards smart city [1-3].

Provision of street lighting infrastructure is one of the ways to lessen the rate of road accidents [4]. Provision of street lighting requires high cost since street lighting management need to be very efficient. As mentioned in [7], smart street lighting contributes to energy savings whereby light dimming can lower $40 \%$ of the energy used and saves $50 \%$ of maintenance cost and prolonged lamp life to $25 \%$ longer as summarized by Table 1 .

Table 1. LED Activity and Saving

\begin{tabular}{ll}
\hline LED Activity & Benefit \\
\hline \multirow{2}{*}{ Remote ON/OFF and dimming of lights } & $40 \%$ of energy saving \\
& $50 \%$ of light maintenance cost \\
& Prolong lamp life 25\% \\
\hline
\end{tabular}


Currently many street lighting, which is fixed to be $\mathrm{ON}$ in the evening and OFF before the sun set [6] without taking into consideration the ambient brightness and also the number of vehicles. Figure 1 shows the real condition on a street in Klang Valley area in Malaysia at 4:30 pm but it was very cloudy and dark. However, the street lights are still OFF. This situation provides less comfort to motorist.

Additionally, switching on the lights increases the use of energy. Thus, Ambient Street Lighting System was developed to control the ON/OFF street lighting based on the ambient brightness, i.e. weather and the number of vehicles, while providing safety for the road users.

Ambient Street Lighting is a prototype to control automatically the street lights based on hybrid factors i.e. ambient brightness and numbers of vehicles on the road. Ambient brightness factors include the change of day and night as well as cloudy weather that contributes to dark atmosphere even though it is daytime.

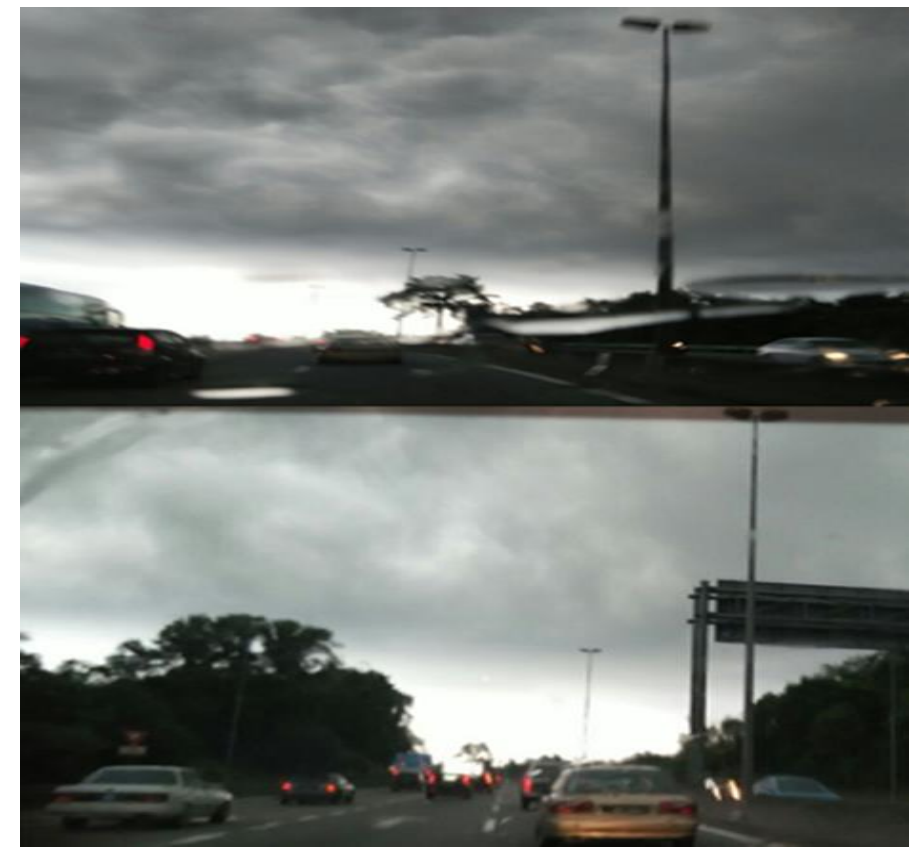

Figure 1. The Real Situation At A Street During Daytime

The remainder of this work is organized as follows. Section II describes the related works that have already been implemented. Section III describes the system overview and the block diagram of this project. Finally, Section IV discusses prototype testing and validation. Section V concludes this paper and discusses directions for potential future work.

\section{RELATED WORKS}

Many methods previously proposed for street lighting such as using a GSM technique to remotely control the street lights [5]. This technique uses LDR installed on the streets lighting to detect day/night as well as the lamp life. Hence, the street lights are switched ON based on the brightness and darkness of day and night. The technique in this paper also monitors the lamp life so immediate action can be taken if the lights are damaged. In [7], proposed a method to automatically control street lightings using LDR sensor to detect street intensity in real time for energy saving. In [8], developed a prototype to provide a smart street lighting by using LED luminaries through communication circuits to send/receive data from the central node and LED sensors. This is an IOT based project to save energy and ease of control of street lighting [9].

\section{AMBIENT STREET LIGHTING DESIGN}

Ambient Street Lighting is an alternative way to reduce the power consumption in the streets and make the street lighting system more efficient. It is a prototype that automatically control lighting at the street based on ambient intensity and also number of passing by vehicles on the road. Ambient intensity condition 
is detected by using LDR sensors while street traffic is monitored using photoelectric sensors. Hence, street lighting is $\mathrm{ON}$ if the surrounding is dark and brightness is improved if fewer vehicles passing by to provide safer surroundings. While the brightness level of the street lights will be dimmed in darker surroundings having large number of vehicles passing by. This is the factor to saves energy as the streets will be lit up with the vehicles lamp. Hence, this technique saves more energy as the light is ON based on needs.

The components for the proposed system have been classified into a few components group; input, output and controller. Two type of input used in this system; power supply and sensor. The power supply is meant to provide electricity power for the overall system while the sensor is used to sense dark and light surrounding. Two types of sensors were used, i.e. Light Depends Resistor (LDR) and Ultrasonic Distance Sensor (UDS), i.e. for surrounding intensity sensing and to detect the number of cars passing by the street, respectively. Next, Arduino UNO Atmega 328P-PU has been selected as the controller in this system due to the low cost, compatibility, compact size and easy interfacing over several type of other controller including Field Programmable Gate Array (FPGA), Programmable Logic Controller (PLC) and Programmable Integrated Circuit (PIC).

Light intensity are the output used in the proposed system and connected to the microcontroller. The LED module represents the street light and the intensity module controls the brightness of the LED. Three level of brightness value have been included in the intensity module as a response to the condition and sensor input including the street lamp period. As for the software part, Arduino Programming has been used for the Arduino Microcontroller to instruct the program of the prototype. The block diagram of the proposed prototype is portrayed by Figure 2 .

The system is operated based on two main factors i.e. surrounding brightness and number of cars. In our experiment, the calculation of the number of cars are to simulate enhanced brightness from car lights. Hence, during day light the high intensity will reduce/dimmed or OFF the light.

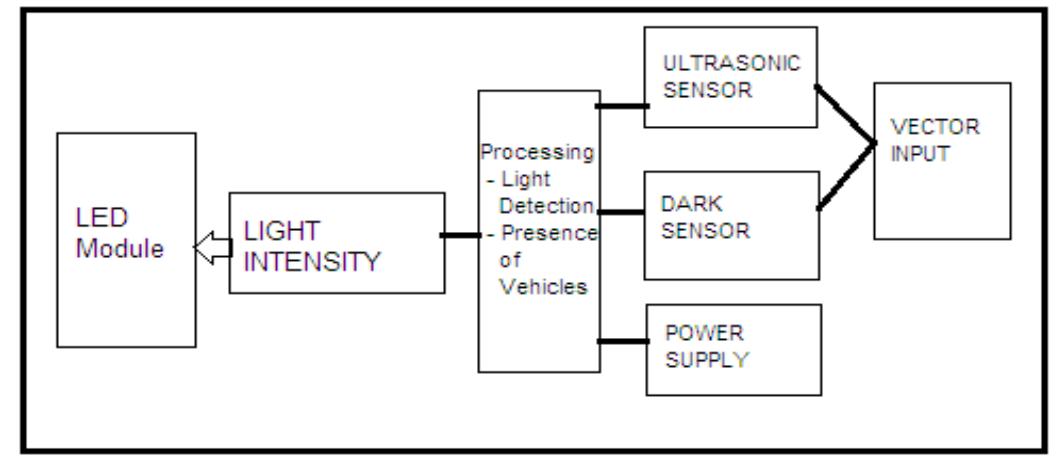

Figure 2. A Block Diagram of the Street Lighting Prototype

The system is operated based on two main factors i.e surrounding brightness and number of cars. In this case, the calculation of the number of cars are to simulate enhanced brightness from car lights. Hence, during day light the high intensity will reduce/dimmed or OFF the light. During the night time, low intensity will ON the lights. However, if number of cars increases, the street lighting brightness will be reduced based on number of cars.

Figure 3 shows the flowchart of the designed system. Firstly, the system will start with detecting the brightness of the surrounding environment, if the value is more than the threshold value, then the street light will turn $\mathrm{ON}$ with low intensity. Then, as a trigger it will start counting how many traffic users on that current time, if there is no vehicle passing by the road, then it will go back to default brightness which is the low intensity. If the sensor detect the traffic at the roadways is not busy, only few vehicle passing by the road, the brightness of the street light will become high intensity then if the sensor detect the road is busy, it will send the signal to microcontroller to provide medium intensity of the street light brightness.

In order to set the threshold values, a few intensity readings were taken based on surrounding darkness and brightness as summarized by Table 2. The threshold value is set to 500 based on the weather conditions and surroundings situation. While for number of cars, the threshold value is set to 3 in the lab experiment. For the lab testing, we decided 3 cars could be enough to simulate the surrounding brightness.

All the components mentioned earlier were connected to each other using embedded-based program. Figure 4 depicted the final setting of the complete prototype. 


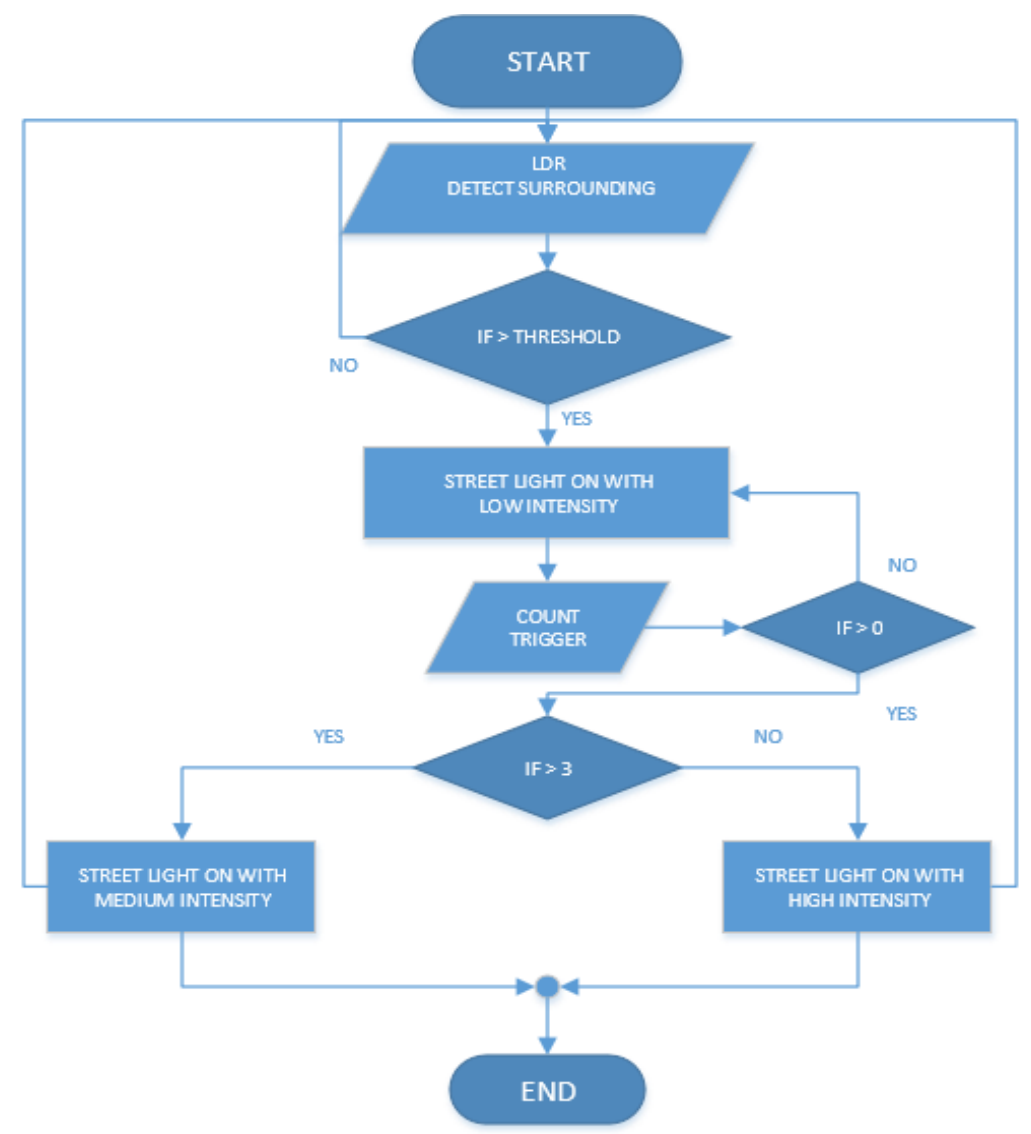

Figure 3. A Flow Diagram of the Street Lighting Prototype

Table 2. Reading of surrounding darkness and brightness intensity

\begin{tabular}{llll}
\hline Time $(\mathrm{am} / \mathrm{pm})$ & Ambient Condition & Intensity $(\mathrm{V})$ & Light Response \\
\hline 8.00 a.m & Sunny & 36 & OFF \\
9.00 a.m & Sunny & 30 & OFF \\
12 p.m & Sunny & 20 & OFF \\
4 p.m & Sunny & 60 & OFF \\
6 p.m & Sunny & 50 & OFF \\
12 p.m & Rainy & 207 & OFF \\
15 p.m & Rain and Cloudy & 295 & OFF \\
16 p.m & Rain and Dark & 500 & ON \\
1850 p.m & Dusky & 905 & ON \\
2000 p.m & Very dark & 994 & ON \\
\hline
\end{tabular}

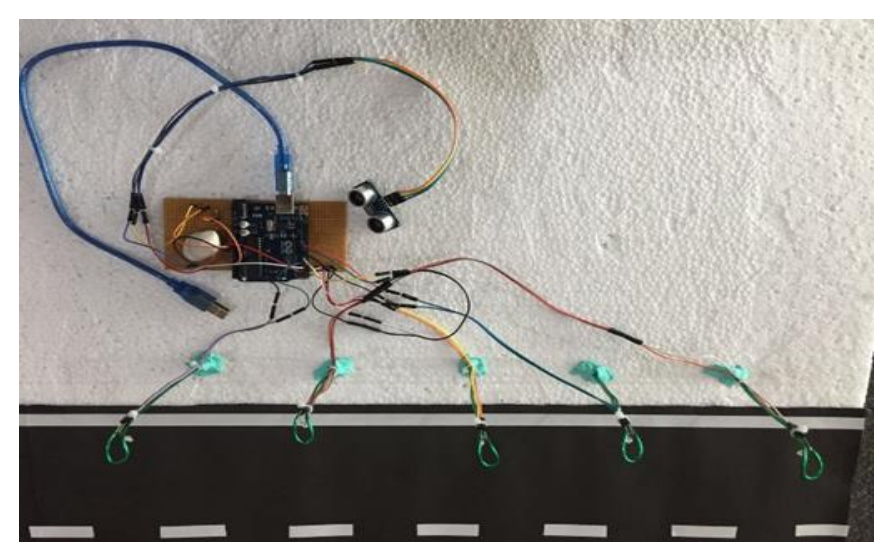

Figure 4. The Complete Prototype Setup 


\section{SYSTEM TESTING AND VALIDATION}

Test conducted on connected and calibrated completed prototype. LDR is covered with cork to test LDR capability for dark surroundings. For this test, there are no vehicles on the road. As a result, as shown in Figure 5 the street lights model will be ON with low intensity. The brightness level is lowered due to energy saving as there is none any vehicles passing by on the street at that time. Experimental Results of Uniform Random-3-SAT Benchmark Instances.

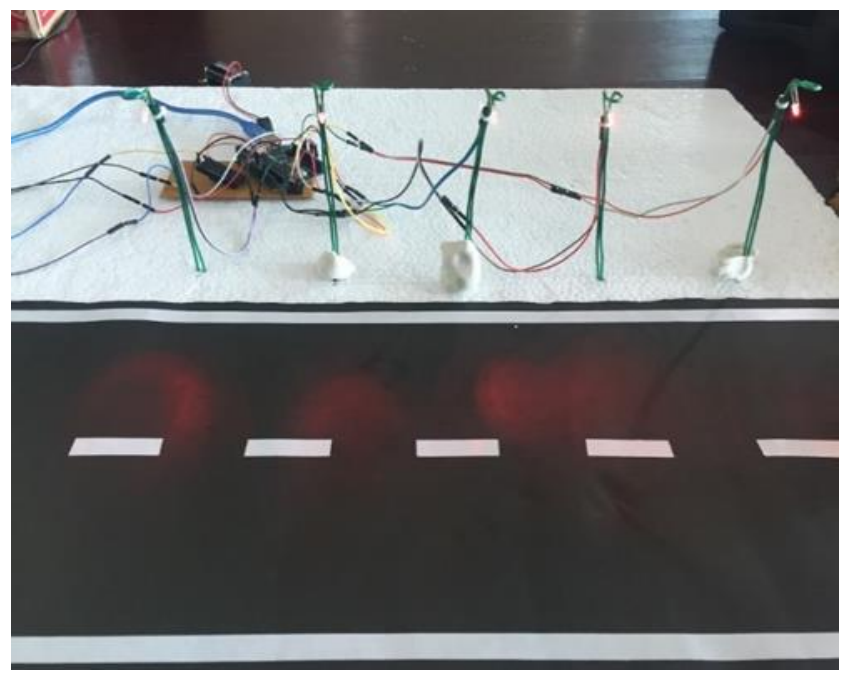

Figure 5. Street Light ON with Low Intensity

In Figure 6, LDR is closed to simulate dark surroundings but 5 cars are used on the streets of the prototype. Even though the value of intensity is 990 , but UDS detected medium intensity due to the existence of a number of cars on the street. As such, the streets lights are ON with medium intensity as the surroundings have been lit up with the cars light.

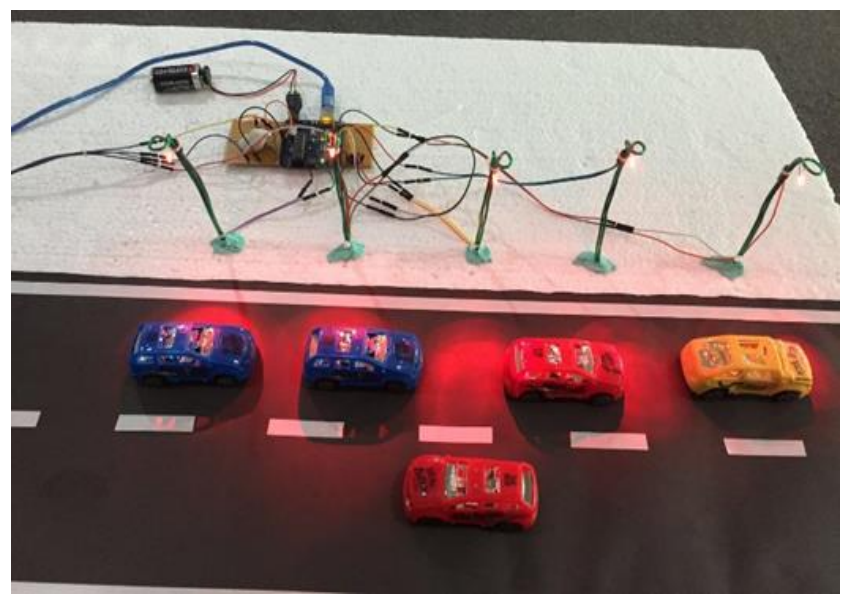

Figure 6. Street Light ON with Medium Intensity

In Figure 7, we tested for full darkness, with 990 intensity, but with 3 number of cars. The light intensity is at high due to the fact that it is supposed to light up the street for the motorists passing by. In our, experiment, we only conduct tests for the capability of the LDR and UDR to detect light intensity and number of cars in order for the street lights to be ON and OFF. We do not conduct any test from the prototype for maintenance cost and energy saving as well as the lamp life. It will be considered in the future phase of the research. 


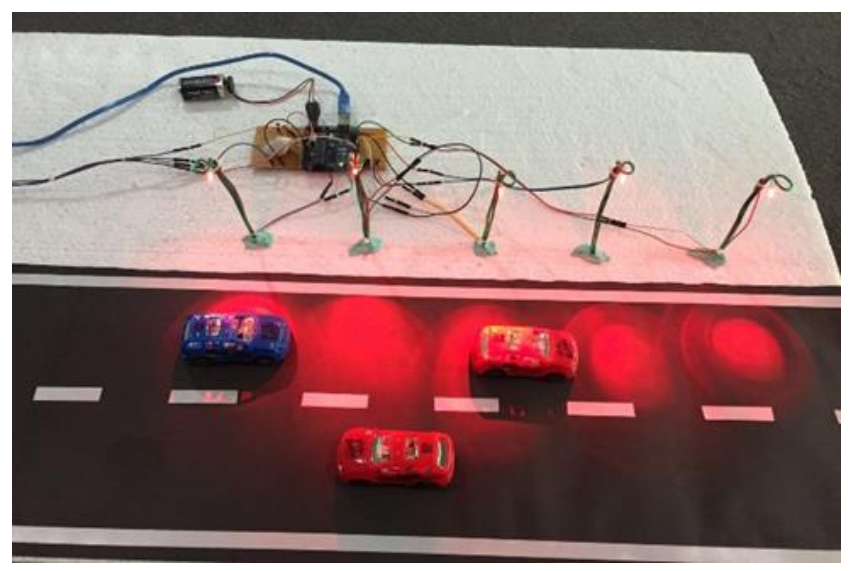

Figure 7. Street Light ON with High Intensity

\section{CONCLUSION}

This street lights prototype based on surrounding factors have been developed and tested with a few scenarios i.e. dark, semi-dark, and bright. Plus with some number of cars to show the capability of the sensor detecting surrounding light intensity with number of cars passing by on the street. Street lights have reacted with success towards surrounding brightness/darkness and number of cars passing by on the street. The prototype can be improved further by considering the energy saving and maintenance cost effect for future research. In general, this technique is usable on the streets as a good improvement for the existing infrastructure towards smart city and energy saving as well as for road users' comfort and safety.

\section{ACKNOWLEDGEMENTS}

Abidin.

This research is partially funded by University Tenaga Nasional and Universiti Sultan Zainal

\section{REFERENCES}

[1] "Royal Society for the Prevention of Accidents -Street Lighting and Road Safety". Available at http://www.rospa.com/road-safety/advice/roads/street-lighting/

[2] Steinbach R, Perkins C, Tompson L, Johnson S, Armstrong B, Green J, Grundy C, Wilkinson P, Edwards P. “The effect of reduced street lighting on road casualties and crime in England and Wales: controlled interrupted time series analysis". J Epidemiol Community Health. 2015; 69(11):1118-1124.

[3] Ghazwan A. "The Impact of New Street Lighting Technologies on Traffic Safety". Journal of Traffic and Logistics Engineering. 2014; 2(3):202-205.

[4] Jaafar T R, Mustafa M F, Kemin S, Kasiran R. "Kemalangan Jalan Raya: Analisis Data Membabitkan Pengguna Motosikal". Jurnal Teknologi. 2013; 38(B):1-14.

[5] Rajput K Y, Khatav G, Pujari M, Yadav P. "Intelligent Street Lighting System Using GSM". International Journal of Engineering Science Invention. 2013; 2(3): 60-69.

[6] Yusnani M Y, Roszainiza R, Mohd U K, Mustaffa S. "Towards Smart Street Lighting System in Malaysia". In Proc. IEEE Symposium on Wireless Technology and Applications (ISWTA), September 22-25, 2013, Kuching, Malaysia.

[7] Derek Markham, "Global Warming Effects and Causes: A Top 10 List. Available at http://planetsave.com/2009/06/07/global-warming-effects-and-causes-a-top-10-list/

[8] Ruchitha K S, Agarwal N, Anand S, Das A, Rajasree P M. "Design and Development of Automatic Adjustment of Street Light Intensity". International Journal of Advanced Research in Electrical, Electronics and Instrumentation Engineering. 2016; 5(4):2717-2721.

[9] Eshika S, Vaibhav S, Dr. Annapurna D. "Remotely Controlled Automated Street Lights: A Novel Approach towards IoT (Internet of Things)". In Proc. Recent Innovations in Science and Engineering (RISE 2016) 30th April 2016, Bangalore, India. 